\title{
Feasibility and efficacy of simultaneous integrated boost intensity-modulated radiation therapy in patients with limited-disease small cell lung cancer
}

\author{
Dan Han ${ }^{1,2}$, Qin Qin', Shaoyu Hao ${ }^{3}$, Wei Huang ${ }^{1}$, Yumei Wei ${ }^{1}$, Zicheng Zhang ${ }^{1}$, Zhongtang Wang
} and Baosheng Li ${ }^{1 *}$

\begin{abstract}
Purpose: To evaluate the feasibility and efficacy of simultaneous integrated boost intensity-modulated radiation therapy (SIB-IMRT) in patients with limited-disease small-cell lung cancer (LD-SCLC).

Methods: Patients with LD-SCLC were treated with SIB-IMRT within 1 week after completion of 2 cycles of induction chemotherapy. Then 2-4 cycles of adjuvant chemotherapy were administered within 1 week after SIB-IMRT. Irradiation was given accelerated hyper-fractionated with the prescribed dose 57Gy at 1.9Gy twice daily to the gross tumor volume (GTV) , 51Gy at 1.7Gy twice daily to the clinical tumor volume (CTV) and 45Gy at 1.5Gy twice daily to the planning target volume (PTV). The chemotherapy regimen consisted of platinum plus etoposide. Prophylactic cranial radiation (25Gy in 10 fractions) was administered to patients who got complete response (CR) or near complete response $(\mathrm{nCR})$. The primary endpoint of this study was the frequency of grade 3 or higher acute non-hematologic treatment-related toxicities. Secondary end points included objective response, overall survival (OS), progression-free survival (PFS), locoregional recurrence-free survival (LRFS).
\end{abstract}

Results: A cohort of 35 patients were enrolled in the study, the biological equivalent dose (BED) of the GTV in the SIB-IMRT was 59.16Gy. Grade 1, 2, and 3 esophagitis were observed in 11 (31\%), 12 (34\%), and 6 (17\%) patients, respectively; Grade 1 and 2 pneumonitis were observed in 8 (23\%) and 4 (11\%) patients, respectively. The median OS and PFS of the whole group were 37.7 months and 29.3 months, respectively. The 1- and 2-year OS was $94.1 \%$ and $68.5 \%$, respectively. The 1- and 2-year PFS was $76.8 \%$ and $40.7 \%$, respectively. The 1 - and 2-year LRFS was $87.7 \%$ and $73.8 \%$, respectively.

Conclusions: SIB-IMRT was feasible and well-tolerated in patients with LD-SCLC, and worth further evaluating in a large prospective clinical trial.

Keywords: Small-cell lung cancer, Lung neoplasms, Limited-stage, Simultaneous boost intensity-modulated radiotherapy, Outcome assessment

\section{Introduction}

Small cell lung cancer (SCLC) accounts for $10 \%-15 \%$ of all lung cancer cases [1]. At the time of diagnosis, 30\%-40\% of SCLC patients present with limited disease (LD) which is defined as disease confined to the hemithorax and the regional lymphatic nodes according to the Veterans Administration Lung Study Group staging [2].

\footnotetext{
* Correspondence: baoshli1963@163.com

1 Department of 6th Radiation Oncology, Cancer Prevention and Treatment Institute of Shandong Province, Shandong 250117, China

Full list of author information is available at the end of the article
}

The combination of thoracic radiotherapy (TRT) with chemotherapy has been shown to be the standard treatment for limited-disease small-cell lung cancer (LDSCLC) with improved local control and overall survivals based on two meta-analyses in the 1990s [3,4]. TRT with concurrent chemotherapy was endorsed by many studies because of better local control and longer survival although accompanied with higher toxicities compared with sequential chemoradiothearpy. 
TRT is an indispensable treatment for LD-SCLC, however, the optimal radiotherapy approach remains controversial with respect to timing, dose-fractionation, and target definition. As far as dose-fractionation is concerned, accelerated hyper-fractionated radiotherapy (45Gy with $1.5 \mathrm{~Gy}$ twice daily in 3 weeks) and dose-escalated conventional radiotherapy (60-70Gy with 2 Gy once daily in 6 to 7 weeks ) have been documented as reliable schedules, and an international randomized trial is ongoing to compare these two schedules concurrent with chemotherapy in the treatment of LD-SCLC [5].

Despite of high sensibility to chemotherapy and radiotherapy, SCLC is characterized by inevitable local recurrence and distant metastasis due to its aggressive nature. As a dose-response relationship exists in treating LDSCLC, it is reasonable to apply appropriately high doses to adequate target volumes as long as increased toxicities are acceptable to achieve better local control and subsequently longer survival [6]. Komaki et al. [7] found that high-dose thoracic radiation given twice daily during cisplatin-etoposide (EP) chemotherapy for LD-SCLC improved the rates of local control. Simultaneous integrated boost intensity-modulated radiotherapy (SIBIMRT), in which different dose prescriptions can be delivered simultaneously to different target volumes in the same treatment fraction, has been advocated in recent years as a dose intensification technique, in which the overall treatment time was reduced, but boosted doses to corresponding volumes were produced [8-11].

To investigate the feasibility and effectiveness of SIBIMRT as a dose intensification technique for patients with LD-SCLC, we conducted a single-center, open-label, and prospective phase II clinical study. The primary objectives of the study were to determine the safety and tolerability of SIB-IMRT in combination with chemotherapy. The secondary objectives included response rate, local control rate, and survival rate of SIB-IMRT in combination with chemotherapy.

\section{Methods and materials \\ Eligibility criteria}

The inclusion criteria included: Eastern Cooperative Oncology Group (ECOG) performance status of 0-2 grade; life expectancy $\geq 6$ months; less than 75 years; no chemotherapy or radiotherapy prior to the study; no serious complications, such as hypertension, coronary heart disease, and psychiatric history; hemoglobin $\geq 100 \mathrm{~g} / \mathrm{L}$; white blood cell count $\geq 3.5 \times 10^{9} / \mathrm{L}$; neutrophil count $\geq$ $1.5 \times 10^{9}$; platelet count $>100 \times 10^{9} / \mathrm{L}$; serum creatinine $\leq 1.5 \times$ the upper limit of normal (ULN); serum bilirubin $\leq 2.5 \times$ ULN; Glutamic oxalacetic transaminase and glutamic-pyruvic transaminase $\leq 2.5 \times \mathrm{ULN}$; and alkline phosphatase $\leq 2.5 \times$ ULN.

\section{Exclusion criteria}

The exclusion criteria were as follows: known distant metastasis; history of carcinoma other than SCLC; other serious diseases such as myocardial infarction in the last 6 months; participation in other clinical trials in the last 4 weeks or at present; use of other anti-cancer drugs; and a history of organ transplantation. The study was performed after obtaining patients' consent and under protocols approved by the institutional review boards of the Shandong Cancer Prevention and Treatment Research Ethics Committee.

\section{Pre-treatment evaluation}

Patients were required to undergo a complete medical history before enrollment in the study. To exclude distant metastases, the pre-treatment assessment included a bone scan and computed tomography (CT) scan of the head, neck, chest, and abdomen. A physical examination, electrocardiogram, complete blood count, urinalysis, chemistry tests (including liver and kidney function tests), electrolytes, coagulation panel, and tumor markers were also required. Disease staging was performed according to the American Joint Committee on Cancer (AJCC version 7.0).

\section{Chemotherapy}

The patients were treated with 2 cycles of induction chemotherapy, followed by TRT within 1 week after completion of the second cycle of chemotherapy. Then 2-4 cycles of adjuvant chemotherapy were administered within 1 week after completion of TRT. Chemotherapy consisted of etoposide (100 mg/m2 intravenously on days 1-5) and cisplatin (25 mg/m2 intravenously on days 1-3) and was administered every 3 weeks.

\section{Radiotherapy}

The patients were immobilized using plastic mesh mask in the supine position and then, consecutively underwent four-dimensional computed tomography (4DCT) scanning under free breathing conditions on a 16-slice CT scanner (Philips Brilliance Bores CT). During the scanning procedure, respiratory signals were recorded using the Varian Real-Time Position Management respiratory gating hardware. After 4DCT scanning, the 4D software read all reconstructed images along with the respiratory phases. Images were evenly sorted into 10 phases distributed over a breathing cycle and all CT images were imported into the treatment planning system (Eclipse 8.6, Varian Medical Systems, Palo Alto, CA).

The targets were delineated according to the following criteria. Gross tumor volume (GTV) was manually delineated on all 10 phases of the 4DCT scan. The GTV referred to the restaging chest CT obtained after induction chemotherapy, including the residual primary tumor and 
all clinically-involved lymphatic regions. When enlarged lymph nodes resolved after induction chemotherapy, the previously involved lymph node regions were still included in the radiation target by reviewing the pre-chemotherapy CT scan. Elective treatment of clinically uninvolved lymphatic regions was not carried out. The clinical tumor volume (CTV) was defined by the expanding GTV with a $0.5 \mathrm{~cm}$ margin and the planning target volume (PTV) was defined by the expanding CTV with a $0.5 \mathrm{~cm}$ margin. The prescribed dose was 57Gy in 30 fractions at $1.9 \mathrm{~Gy}$ per fraction to the GTV, 51Gy in 30 fractions at $1.7 \mathrm{~Gy}$ per fraction to the CTV, and $45 \mathrm{~Gy}$ in 30 fractions at $1.5 \mathrm{~Gy}$ per fraction to the PTV.

All fractional doses were given twice daily with at least $6 \mathrm{~h}$ between fractions and 5 times each week. All plans aimed to cover at least $95 \%$ of the PTV with the $90 \%$ isodose, to have minimum dose $>90 \%$ and maximum dose $<110 \%$. The dose-volume histogram (DVH) constraints of the organs at risk (OARs) were as follows: mean lung dose $<20$ Gy and lung V20 < 33\%, spinal cord Dmax $\leqq$ $45 \mathrm{~Gy}$, mean heart dose $<30 \mathrm{~Gy}$ and heart $\mathrm{V} 40<46 \%$, mean esophagus dose < 34Gy, esophagus V35 < 50\% [12,13].

The patients were treated based on a twice-daily threedimensional cone beam computed tomography (CBCT) anatomy registration. $\mathrm{CBCT}$ images were acquired using a scanner attached to the gantry of the Trilogy Linear Accelerator (Varian Medical Systems). The discrepancy between planned and actual tumor position was automatically evaluated based on the automated alignment software. When the quality of a known parameter (such as bony landmarks in the chest) was ambiguous, the tumor contour was manually aligned to verify the automatic alignment results.

The biological equivalent dose (BED) was calculated using the linear quadratic formula: $\mathrm{BED}=(\mathrm{nd})[1+\mathrm{d} /(\alpha / \beta)]-$ $(0.693 t / \alpha$ Tpot $)$, where $\mathrm{n}=$ the total number of fractions delivered; $d=$ the dose per fraction (Gy); $\alpha / \beta=10$ for acute effects and tumor control and 3 for chronic effects; $\alpha=0.3 \mathrm{~Gy}^{-1} ; \mathrm{t}=$ total days in which radiotherapy is delivered; and Tpot=potential doubling time (5.6 days) [14].

\section{Prophylactic cranial irradiation ( $\mathrm{PCl}$ )}

After completion of chemotherapy and TRT, patients who achieved a complete response (CR) or near complete response (nCR) were offered the option of PCI. Patients were administered PCI (25Gy in 10 fractions to the entire brain) within 4 weeks after completion of all chemotherapy.

\section{Adverse effect assessment}

Side effect assessment was graded at least weekly using the National Cancer Institute Common Toxicity Criteria (version 3.0) during radiotherapy and chemotherapy period. 3 months after the treatment, late toxicities were evaluated according to the Radiation Therapy Oncology Group (RTOG)/European Organization for Research and Treatment of Cancer late radiation morbidity scoring schema.

\section{Follow-up}

The treatment response was estimated using CT or positron emission tomography/computed tomography after treatment, according to the Response Evaluation Criteria in Solid Tumors (version 1.0). Follow-up after treatment completion was every 3 months over the first 2 years and every 6 months thereafter. Each visit included medical history, physical examination, complete blood count, chest and abdomen $\mathrm{CT}$, brain magnetic resonance imaging/CT, and bone scan (if necessary).

\section{Study endpoints and statistics}

The primary endpoint of this study was the frequency of grade 3 or higher acute non-hematologic treatmentrelated toxicities. Secondary end points included objective response, overall survival (OS), progression-free survival (PFS), locoregional recurrence-free survival (LRFS). OS was observed from the first day of treatment until death or last follow-up time, PFS was observed from the first day of treatment until progress, death or last follow-up time, and LRFS was observed from the first day of treatment until recurrence, death or last follow-up time. The Kaplan-Meier method was used to estimate OS, PFS and LRFS using SPSS ${ }^{\curvearrowleft}$ v. 17.0 (SPSS Inc, Chicago, IL).

\section{Results}

\section{Patient characteristics}

Between AUG, 2009 and FEB, 2013, 44 SCLC patients in Shandong Cancer Hospital \& Institute were enrolled in the study. 9 patients were excluded from the analysis of eligible patients, and leaving 35 assessable patients. The reasons for ineligibility were extensive disease (3), incomplete staging studies (1), absence of study data (2) and lose of follow up (3). All of the 35 patients finished the planned induction chemotherapy, radiotherapy, and adjuvant chemotherapy. 22 patients were given PCI within 4 weeks after completion of all chemotherapy. Of the 35 patients, 14 patients had stage IIIA, 15 had stage IIIB,4 had stage IIA, 1 had stage IIB, and 1 had stage IA. The median age was 54 years (range, $35-72$ years). The patients' characteristics were summarized in Table 1.

\section{Radiotherapy plan evaluation}

The BED of the GTV in the SIB-IMRT was 59.16Gy. The evaluation of the DVH-based parameters of the OARs were shown in Table 2. 
Table 1 Characteristics of 35 patients

\begin{tabular}{ll}
\hline Characteristic & Number of cases (\%) \\
\hline Age (y) & $54(35-72)$ \\
Gender & $26(74 \%)$ \\
Male & $9(26 \%)$ \\
Female & \\
ECGO performance status & $12(34 \%)$ \\
0 & $22(63 \%)$ \\
1 & $1(3 \%)$ \\
2 & \\
T-stage (AJCC 7) & $4(11 \%)$ \\
1 & $9(26 \%)$ \\
2 & $10(29 \%)$ \\
3 & $12(34 \%)$ \\
4 & \\
N-stage (AJCC 7) & $3(9 \%)$ \\
0 & $4(11 \%)$ \\
1 & $23(66 \%)$ \\
2 & $5(14 \%)$ \\
3 &
\end{tabular}

Abbreviations: ECOG Eastern Cooperative Oncology Group.

AJCC American Joint Committee on Cancer, $T$ Tumor, $N$ Node.

\section{Toxicity}

The toxicities for each patient were presented in detail (Table 3). 15(43\%)and 7(20\%)patients had grade 3 and 4 hematologic toxicity, respectively. Necessary treatment measures, such as recombinant human interleukin and granulocyte colony stimulating factor, were provided and blood transfusion were given to the patients with grade 4 hemoglobin toxicity (All patients fully recovered from hematologic toxicity). Of the 35 patients, grade 1,2 , and 3 esophagitis occurred in 11 (31\%), 12 (34\%), and 6 (17\%) patients, respectively; the patients with grade 3 esophagitis required intravenous nutrition. Grade 1 and 2 pneumonitis occurred in 8 (23\%) and 4 (11\%) patients, respectively. None of the patients died of treatment-related causes.

\section{Treatment response}

The efficacy of induction chemotherapy was observed in 35 patients; 29 patients achieved partial response (PR), 4 patients achieved CR and 2 patients had stable disease (SD). 4 weeks after radiotherapy and chemotherapy, of the 35 patients, $51 \%$ of patients $(n=18)$ had CR, $11 \%$ of patients $(\mathrm{n}=4)$ had $\mathrm{nCR}$, and another $26 \%(\mathrm{n}=9)$ had PR, for an overall response rate of $88 \%$; another $9 \%$ of patients $(n=3)$ had SD, 3\% $(n=1)$ had progressive disease (PD), and no one died before the 4 weeks post-treatment response could be evaluated.
Table 2 The DVH-based parameters of the OARs in the study

\begin{tabular}{|c|c|}
\hline & mean $\pm S D$ \\
\hline \multicolumn{2}{|l|}{ Total lungs } \\
\hline $\operatorname{MLD}(G y)^{a}$ & $14.8 \pm 1.7$ \\
\hline V5 $(\%)^{\mathrm{b}}$ & $67.4 \pm 9.0$ \\
\hline V10 $(\%)^{\mathrm{b}}$ & $53.5 \pm 7.2$ \\
\hline V20 $(\%)^{\mathrm{b}}$ & $28.7 \pm 3.2$ \\
\hline V30 $(\%)^{b}$ & $17.1 \pm 2.6$ \\
\hline \multicolumn{2}{|l|}{ Ipsilateral lungs } \\
\hline MLD (Gy) & $22.1 \pm 4.1$ \\
\hline V5 (\%) & $79.1 \pm 10.7$ \\
\hline V10 (\%) & $70.7 \pm 11.8$ \\
\hline V20 (\%) & $47.2 \pm 11.3$ \\
\hline V30 (\%) & $30.3 \pm 10.0$ \\
\hline \multicolumn{2}{|c|}{ Contralateral lungs } \\
\hline MLD (Gy) & $8.8 \pm 2.9$ \\
\hline V5 (\%) & $53.1 \pm 13.1$ \\
\hline V10 (\%) & $32.1 \pm 12.7$ \\
\hline V20 (\%) & $11.8 \pm 8.8$ \\
\hline V30 (\%) & $5.2 \pm 4.6$ \\
\hline \multicolumn{2}{|l|}{ Spinal cord } \\
\hline $\operatorname{Dmax}(G y)^{e}$ & $42.7 \pm 3.7$ \\
\hline \multicolumn{2}{|l|}{ Heart } \\
\hline Dmean $(G y)^{a}$ & $16.1 \pm 7.3$ \\
\hline V30 (\%) & $23.3 \pm 15.0$ \\
\hline V40 (\%) & $9.0 \pm 5.0$ \\
\hline \multicolumn{2}{|l|}{ Esophagus } \\
\hline $\operatorname{MED}(\mathrm{Gy})^{\mathrm{a}}$ & $29.0 \pm 6.7$ \\
\hline V45 (\%) ${ }^{\mathrm{d}}$ & $33.6 \pm 5.3$ \\
\hline
\end{tabular}

Abbreviations: SD standard deviation. ${ }^{\text {T}}$ The mean irradiation dose that the lung, heart and esophagus received, respectively; ${ }^{b}$ the volume of the lung that received the 5, 10,20 and 30Gy irradiation dose, respectively; ${ }^{C}$ the volume of the heart that received 30 and $40 \mathrm{~Gy}$ irradiation dose, respectively; ${ }^{d}$ the volume of the esophagus that received 45Gy irradiation dose; ${ }^{e}$ the maximum irradiation dose that spinal cord received.

\section{Survival}

The median follow-up was 24.6 months, with a range of 6-53.5 months until the last follow-up date (15 August 2014). The median OS and PFS of the entire group were 37.7 and 29.3 months, respectively. The OS, PFS and LRFS were illustrated in Figures 1, 2, and 3, respectively. The 1- and 2-year OS were $94.1 \%$ and $68.5 \%$, respectively. The 1- and 2-year PFS were $76.8 \%$ and $40.7 \%$, respectively. The 1 - and 2 -year LRFS were $87.7 \%$ and $73.8 \%$, respectively. At the last follow-up, 15 (43\%) patients were alive with no evidence of failure. Local regional recurrences only developed in $4(11 \%)$ patients and distant metastases only occurred in $12(34 \%)$ patients. $4(11 \%)$ patients had a 
Table 3 Treatment-related toxicity

\begin{tabular}{lllll}
\hline & \multicolumn{4}{l}{$\begin{array}{l}\text { Grade }(\mathbf{n}=\mathbf{3 5}) \\
\text { of patients }\end{array}$} \\
\cline { 2 - 5 } Category & $\mathbf{1}$ & $\mathbf{2}$ & $\mathbf{3}$ & $\mathbf{4}$ \\
\hline Hematologic toxicity (WBC) & $4(11 \%)$ & $21(60 \%)$ & $9(26 \%)$ & $1(3 \%)$ \\
Hematologic toxicity (PLT) & $4(11 \%)$ & $2(6 \%)$ & $2(6 \%)$ & $3(9 \%)$ \\
Hematologic toxicity (HB) & $8(23 \%)$ & $5(14 \%)$ & $4(11 \%)$ & $3(9 \%)$ \\
Stomach/intestine & $13(37 \%)$ & $11(31 \%)$ & $2(6 \%)$ & 0 \\
Esophagitis & $11(31 \%)$ & $12(34 \%)$ & $6(17 \%)$ & 0 \\
Pneumonitis & $8(23 \%)$ & $4(11 \%)$ & 0 & 0 \\
Weight loss & $3(9 \%)$ & $1(3 \%)$ & 0 & 0 \\
Skin (within the irradiated field) & $2(6 \%)$ & 0 & 0 & 0 \\
Fever & $1(3 \%)$ & $3(9 \%)$ & 0 & 0 \\
\hline
\end{tabular}

Abbreviations: $W B C$ white blood cell, $P L T$ platelet, $H B$ hemoglobin.

local recurrence and distant metastases simultaneously. Among the 16 patients who developed distant metastases, the most common sites were brain $(n=6)$, followed by liver $(n=3)$, adrenals $(n=2)$, lung $(n=2)$, bone $(n=1)$, subcutaneous tissue $(\mathrm{n}=1)$ and supraclavicular lymph nodes $(n=1)$.

\section{Discussion}

The best method of integrating thoracic radiation with chemotherapy includes conflicting reports. Turrisi et al. [15] conducted a randomized trial that demonstrated a twice daily regimen of 45Gy/30 fractions over 3 weeks that produced an acute grade 3 esophagitis rate of $27 \%$, a local regional failure rate of $36 \%$, and a 2 -year OS rate of $47 \%$ was superior to $45 \mathrm{~Gy}$ in 25 daily fractions. The NCCTG 95-20-53 trial [16], which included 6 cycles of EP and cycles 4 and 5 included concurrent chemotherapy and TRT (30Gy/20 twice daily fractions, a 2-week break, and another 30Gy/20 twice daily fractions), resulted in a favorable 5-year survival rate of $24 \%$; however, the locoregional failure remained a problem and grade 3 or grade 3 + toxicity were as high as 97\%. In the RTOG 0239 study [7], patients with LD-SCLC were given thoracic radiation to $61.2 \mathrm{~Gy}$ over 5 weeks (daily $1.8 \mathrm{~Gy}$ fractions on days 1 22 , then twice daily $1.8 \mathrm{~Gy}$ fractions on days 23-33), and the rate of grade 3 esophagitis was $18 \%$ and local regional failure rate was $20 \%$, but the 2 -year OS rate of $36.6 \%$ did not reach the projected goal. A newer method should allow the safe administration of higher doses of TRT.

In the present study, we first applied SIB-IMRT that delivered GTV at 57Gy in 30 fractions, CTV at 51Gy in 30 fractions, and PTV at 45Gy in 30 fractions twice daily over 3 weeks. The dose could be escalated in a single plan for the entire treatment, but still met dose constraints to critical normal structures, such as the heart, lung, esophagus, and spinal cord. Favorable results were achieved, with grade 3 esophagitis rate of $17 \%$, and 2 -year OS of $68.5 \%$, and 2-year LRFS was $73.8 \%$. Of note, the sample size of the study was small.

Despite concurrent chemoradiotherapy represents the standard treatment for patients with LD-SCLC, we applied sequential chemoradiotherapy in the current trial considering serious toxicity from concurrent chemoradiotherapy and insufficient supportive treatment in developing country [17]; meanwhile, we supposed that sequential

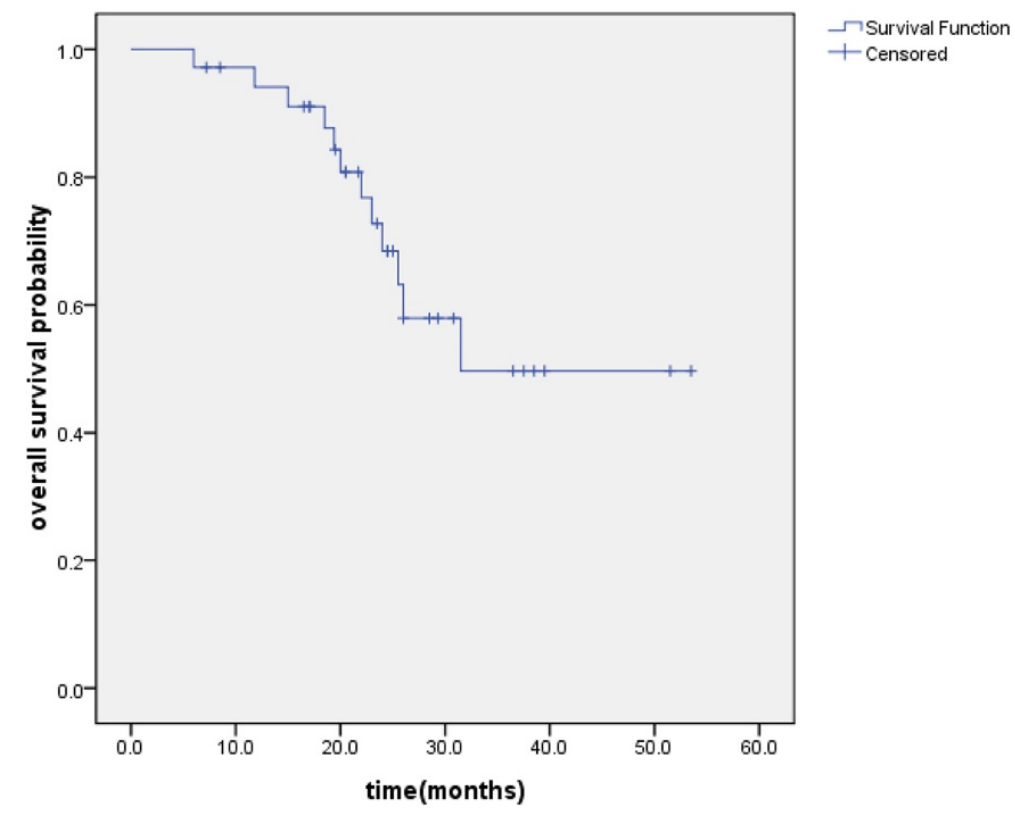

Figure 1 Kaplan-Meier plot of overall survival. 


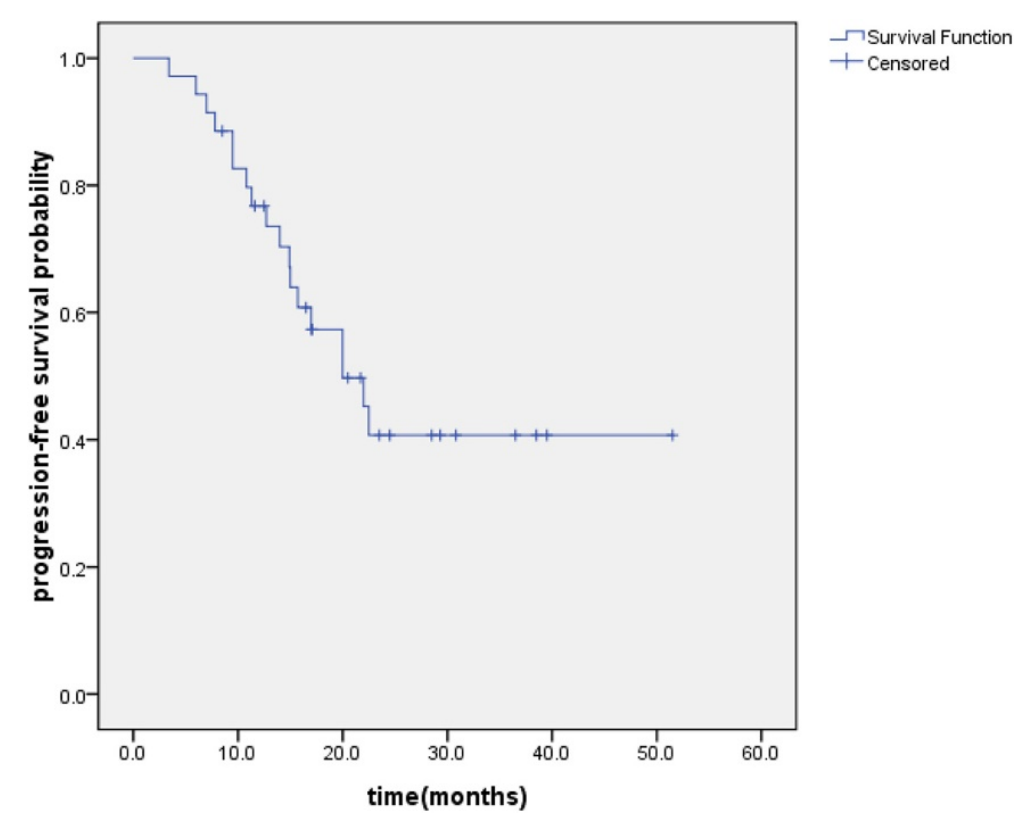

Figure 2 Kaplan-Meier plot of progression-free survival.

chemoradiotherapy may not be inferior to concurrent chemoradiotherapy. SCLC is characterized by the predominantly central type and bulky tumor [1], so hematologic toxicity and esophagitis are more severe in the concurrent regimen. Choi et al. [18] reported that esophagitis limited treatment when total dose from twice daily treatment exceeded 45Gy concurrent chemotherapy. Furthermore, in a phase III study from the Japanese Clinical Oncology Group , 231 patients who received 4 cycles of cisplatin plus etoposide were randomly assigned to either sequential or concurrent twice daily 45 Gy TRT. There was no statistically significant difference $(p=0.097)$ in survival between the two groups [19].

Our results in the 35 patients treated according to the upper approach indicate clear feasibility. The main toxicity problems of our study were grade 3 esophagitis and grade 2 pneumonitis, affecting $17 \%$ and $11 \%$, respectively. The $17 \%$ severe acute esophagitis rate in this study was higher than the $4 \%$ experienced in the sequential arm of Japanese Clinical Oncology Group Study 9104 because of the

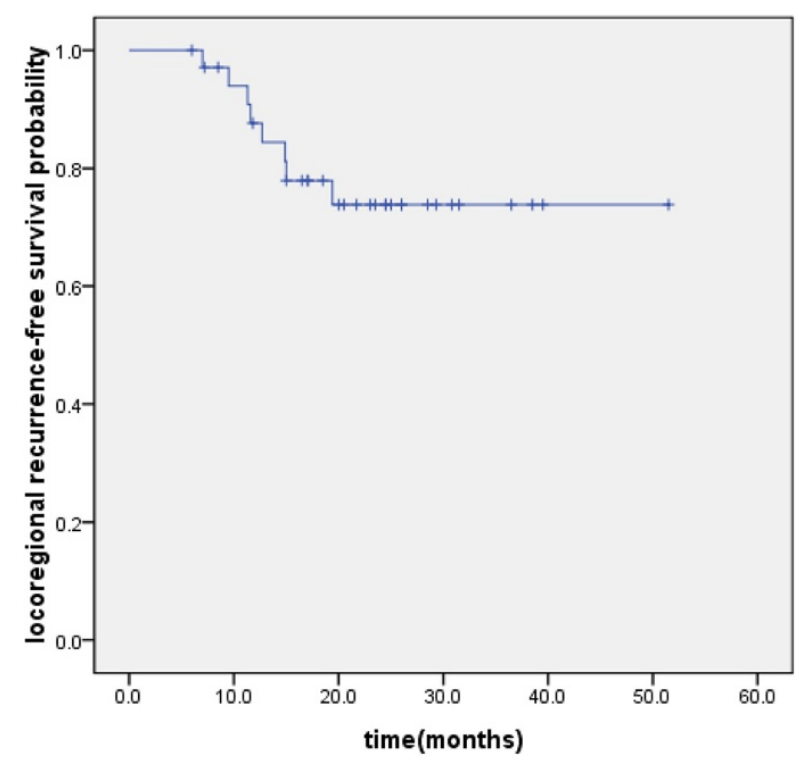

$\neg$ Survival Function

+ Censored

Figure 3 Kaplan-Meier plot of locoregional recurrence-free survival. 
higher radiation dose [19]. Esophagitis after SIB-IMRT did not lead to limitation, and all the affected patients recovered their ability to swallow. The grade 3 esophagitis rate was lower than the $27 \%$ occurred in the twice daily arm of INT 0096. The INT 0096 protocol called for starting thoracic radiation on day 1 of chemotherapy on the basis of other studies showing that local control and survival were better when the radiation was started early relative to the chemotherapy $[20,21]$. The TRT in this study began within 1 week after completion of the $2^{\text {nd }}$ cycle of chemotherapy, which may allow us to reduce the volume of esophagus within the radiation fields and synergetic toxicity during therapy.

Thoracic RT affects the patient outcome by decreasing the tumor burden within the chest, resulting in enhanced local control and survival [3,4]. Despite the addition of thoracic RT to chemotherapy, local treatment failures occur in approximately one third of patients treated with the currently accepted optimal therapy in LD-SCLC [16]. Recent evidence supports the hypothesis that TRT dose intensification improves LD-SCLC patients' outcomes [6]. Our results supported this view and showed a favourable outcome that the 2-year LRFS was $73.8 \%$, which was in agreement with the data reported by RTOGP0239 [7].

As a dose-response relationship exists in treating LDSCLC, local control and subsequent survival are associated with dose-fractionation parameters [13]. The 2-year OS rate of $68.5 \%$ in the present study was higher than the $47 \%$ in the twice-daily arm of INT 0096 and the $36.6 \%$ in the RTOGP0239. Compared to the INT 0096, we got a higher BED of 59.16Gy to the tumor with the SIB-IMRT. The BED can be used to compare the efficacy of various dose-fractionation regimens in providing tumor control and survival [22,23].

Distant metastasis was the dominant cause of failure and the most common site was brain (17\%) in present study. Several meta-analyses and randomized trials have focused on the role of PCI in patients with SCLC. A metaanalysis [24] involving 987 patients with SCLC and a CR to chemotherapy showed a $5.4 \%$ increase in the 3-year survival for those undergoing PCI. Although Tai et al. [25] reported that patients with a PR benefited from PCI, with a reduced rate and delayed time for development of brain metastases, patients who got $\mathrm{CR}$ or $\mathrm{nCR}$ were given $\mathrm{PCI}$ in this study. 22 patients were given $\mathrm{PCI}$ and there were 10 patients in whom disease progressed after PCI (3 [14\%] with brain metastases), which was higher than Tai et al. reported (9\%).

In conclusion, the regimen was safe and well-tolerated, and demonstrated an encouraging outcome in patients with LD-SCLC. But this study based on small sample size and further randomized studies should be carried out.

\section{Abbreviations}

SCLC: Small cell lung cancer; LD: Limited disease; TRT: Thoracic radiotherapy; LD-SCLC: Limited disease small cell lung cancer; EP: Cisplatin-etoposide; SIB-IMRT: Simultaneous integrated boost intensity-modulated radiotherapy; ECOG: Eastern cooperative oncology group; ULN: Upper limit of normal; CT: Computed tomography; AJCC: American joint committee on cancer; 4DCT: Four-dimensional computed tomography; GTV: Gross tumor volume; CTV: Clinical tumor volume; PTV: Planning target volume; DVH: Dose volume histogram; OARs: Organs at risk; CBCT: Cone beam computed tomography; BED: Biological equivalent dose; PCI: Prophylactic cranial irradiation; CR: Complete response; nCR: Near complete response; OS: Overall survival; PFS: Progression-free survival; LRFS: Locoregional recurrence-free survival; PR: Partial response; SD: Stable disease; PD: Progressive disease.

\section{Competing interests}

The authors declare that they have no competing interests.

\section{Authors' contributions}

$\mathrm{BL}$ and $\mathrm{DH}$ contributed significantly to study design and concept. DH also contributed to manuscript writing and study coordinator. ZW, ZZ and YW contributed significantly to the acquisition of data and optimization of treatment plans. SH contributed to statistical analysis. WH contributed to final revision of manuscript. All authors read and approved the final manuscript.

\section{Author details}

${ }^{1}$ Department of 6th Radiation Oncology, Cancer Prevention and Treatment Institute of Shandong Province, Shandong 250117, China. ${ }^{2}$ School of Medicine and Life Sciences, University of Jinan-Shandong Academy of Medical Sciences, Shandong 250062, China. ${ }^{3}$ Department of Thoraic Surgery, Cancer Prevention and Treatment Institute of Shandong Province, Shandong 250117, China.

Received: 30 August 2014 Accepted: 28 November 2014

Published online: 11 December 2014

\section{References}

1. van Meerbeeck JP, Fennell DA, De Ruysscher DK: Small-cell lung cancer. Lancet 2011, 378:1741-1755.

2. Jemal A, Siegel R, Ward E, Hao Y, Xu J, Murray T, Thun MJ: Cancer statistics 2008. CA Cancer J Clin 2008, 58:71-96.

3. Pignon JP, Arriagada R, lhde DC, Johnson DH, Perry MC, Souhami RL, Brodin O, Joss RA, Kies MS, Lebeau B: A meta-analysis of thoracic radiotherapy for small-cell lung cancer. N Engl J Med 1992, 327:1618-1624.

4. Warde P, Payne D: Does thoracic irradiation improve survival and local control in limited-stage small-cell carcinoma of the lung? A meta-analysis. J Clin Oncol 1992, 10:890-895.

5. Bogart J, Masters G, Komaki R, Heymach J: Phase III Comparison of Thoracic Radiotherapy Regimens in Patients With Limited Small Cell Lung Cancer Also Receiving Cisplatin and Etoposide. Current Controlled Trials; 2008. https:// www.clinicaltrials.gov/ct2/show/NCT00632853?

6. Yee D, Hanson J, Butts C, Reiman A, Joy A, Smylie M, Fenton D, Chu Q, Roa W: Phase I dose escalation trial of hypofractionated limited-field external beam thoracic radiotherapy for limited-stage small cell carcinoma of the lung. Radiother Oncol 2010, 96:78-83.

7. Komaki R, Paulus R, Ettinger DS, Videtic GM, Bradley JD, Glisson BS, Langer CJ Sause WT, Curran WJ Jr, Choy H: Phase II study of accelerated high-dose radiotherapy with concurrent chemotherapy for patients with limited small-cell lung cancer: radiation therapy oncology group protocol 0239. Int J Radiat Oncol Biol Phys 2012, 83:e531-e536.

8. Franceschini D, Paiar F, Meattini I, Agresti B, Pasquetti EM, Greto D, Bonomo P Marrazzo L, Casati M, Livi L, Biti G: Simultaneous integrated boost-intensitymodulated radiotherapy in head and neck cancer. Laryngoscope 2013, 123:E97-E103.

9. Wu B, McNutt T, Zahurak M, Simari P, Pang D, Taylor R, Sanguineti G Fully automated simultaneous integrated boosted-intensity modulated radiation therapy treatment planning is feasible for head-and-neck cancer: a prospective clinical study. Int J Radiat Oncol Biol Phys 2012, 84:e647-e653.

10. Deenen MJ, Dewit L, Boot H, Beijnen JH, Schellens JH, Cats A: Simultaneous integrated boost-intensity modulated radiation therapy with concomitant capecitabine and mitomycin C for locally advanced anal carcinoma: a phase 1 study. Int J Radiat Oncol Biol Phys 2013, 85:e201-e207. 
11. Fogliata A, Bolsi A, Cozzi L, Bernier J: Comparative dosimetric evaluation of the simultaneous integrated boost with photon intensity modulation in head and neck cancer patients. Radiother Oncol 2003, 69:267-275.

12. Marks LB, Yorke ED, Jackson A, Ten Haken RK, Constine LS, Eisbruch A, Bentzen SM, Nam J, Deasy JO: Use of normal tissue complication probability models. Int J Radiat Oncol Biol Phys 2010, 76(Suppl 3):S10-S19.

13. Gagliardi G, Constine LS, Moiseenko V, Correa C, Pierce LJ, Allen AM, Marks LB: Radiation dose-volume effects in the heart. Int J Radiat Oncol Biol Phys 2010, 76:S77-S85

14. Liu HH, Wang X, Dong L, Wu Q, Liao Z, Stevens CW, Guerrero TM, Komaki R, Cox JD, Mohan R: Feasibility of sparing lung and other thoracic structures with intensity- modulated radiotherapy for non-small cell lung cancer. Int J Radiat Oncol Biol Phys 2004, 58:1268-1279.

15. Turrisi AT 3rd, Kim K, Blum R, Sause WT, Livingston RB, Komaki R, Wagner H, Aisner S, Johnson DH: Twice-daily compared with once-daily thoracic radiotherapy in limited small-cell lung cancer treated concurrently with cisplatin and etoposide. N Engl J Med 1999, 340:265-271.

16. Schild SE, Bonner JA, Hillman S, Kozelsky TF, Vigliotti AP, Marks RS, Graham DL, Soori GS, Kugler JW, Tenglin RC, Wender DB, Adjei A: Results of a phase II study of high-dose thoracic radiation therapy with concurrent cisplatin and etoposide in limited stage small cell lung cancer (NCCTG 95-20-53). J Clin Oncol 2007, 25:3124-3129.

17. Xia B, Chen GY, Cai XW, Zhao JD, Yang HJ, Fan M, Zhao KL, Fu XL: The effect of bioequivalent radiation dose on survival of patients with limited-stage small-cell lung cancer. Radiat Oncol 2011, 6:50.

18. Choi NC, Herndon JE II, Rosenman J, Carey RW, Chung CT, Bernard S, Leone L, Seagren S, Green M: Phase I study to determine the maximum-tolerated dose of radiation in standard daily and hyperfractionated-accelerated twice-daily radiation schedules with concurrent chemotherapy for limited-stage small-cell lung cancer. J Clin Oncol 1998, 16:3528-3536.

19. Minoru T, Masahiro F, Masaaki K, Takahiko S, Akira Y, Soichiro Y, Yutaka N, Koshiro W, Kazumasa N, Tomohide T, Haruhiko F, Nagahiro S: Phase III study of concurrent versus sequential thoracic radiotherapy in combination with cisplatin and etoposide for limited-stage small-cell lung cancer: results of the Japan Clinical Oncology Group Study 9104. J Clin Oncol 2002, 20:3054-3060.

20. Murray N, Coy P, Pater JL, Hodson I, Arnold A, Zee BC, Payne D, Kostashuk EC, Evans WK, Dixon P: Importance of timing for thoracic irradiation in the combined modality treatment of limited-stage small-cell lung cancer. The National Cancer Institute of Canada Clinical Trials Group. J Clin Oncol 1993, 11:336-344.

21. De Ruysscher D, Pijls-Johannesma M, Bentzen SM, Minken A, Wanders R, Lutgens L, Hochstenbag M, Boersma L, Wouters B, Lammering G, Vansteenkiste J, Lambin P: Time between the first day of chemotherapy and the last day of chest radiation is the most important predictor of survival in limited-disease small-cell lung cancer. J Clin Oncol 2006, 24:1057-1063.

22. Fowler JF: Biological factors influencing optimum fractionation in radiation therapy. Acta Oncol 2001, 40:712-717.

23. Fowler JF: The linear-quadratic formula and progress in fractionated radiotherapy. Br J Radiol 1989, 62:679-694.

24. Aupérin A, Arriagada R, Pignon JP, Le Péchoux C, Gregor A, Stephens RJ, Kristjansen PE, Johnson BE, Ueoka H, Wagner H, Aisner J: Prophylactic cranial irradiation for patients with small-cell lung cancer in complete remission. Prophylactic Cranial Irradiation Overview Collaborative Group. N Engl J Med 1999, 341:476-484.

25. Tai P, Assouline A, Joseph K, Stitt L, Yu E: Prophylactic cranial irradiation for patients with limited-stage small-cell lung cancer with response to chemoradiation. Clin Lung Cancer 2013, 14:40-44.

doi:10.1186/s13014-014-0280-9

Cite this article as: Han et al:: Feasibility and efficacy of simultaneous integrated boost intensity-modulated radiation therapy in patients with limited-disease small cell lung cancer. Radiation Oncology 2014 9:280.

\section{Submit your next manuscript to BioMed Central and take full advantage of:}

- Convenient online submission

- Thorough peer review

- No space constraints or color figure charges

- Immediate publication on acceptance

- Inclusion in PubMed, CAS, Scopus and Google Scholar

- Research which is freely available for redistribution 